\title{
Photoinhibition and photoinhibition-like damage to the photosynthetic apparatus in tobacco leaves induced by pseudomonas syringae pv. Tabaci under light and dark conditions
}

\author{
Dan-Dan Cheng ${ }^{1 \dagger}$, Zi-Shan Zhang ${ }^{2 \dagger}$, Xing-Bin Sun ${ }^{1}$, Min Zhao ${ }^{1}$, Guang-Yu Sun ${ }^{1 *}$ and Wah Soon Chow ${ }^{1,3^{*}}$
}

\begin{abstract}
Background: Pseudomonas syringae pv. tabaci (Pst), which is the pathogen responsible for tobacco wildfire disease, has received considerable attention in recent years. The objective of this study was to clarify the responses of photosystem I (PSI) and photosystem II (PSII) to Pst infection in tobacco leaves.

Results: The net photosynthetic rate (Pn) and carboxylation efficiency (CE) were inhibited by Pst infection. The normalized relative variable fluorescence at the $K$ step $\left(W_{k}\right)$ and the relative variable fluorescence at the J step $\left(V_{J}\right)$ increased while the maximal quantum yield of PSII $\left(F_{\mathrm{v}} / F_{m}\right)$ and the density of $Q_{A}$-reducing PSII reaction centers per cross section (RC/CSm) decreased, indicating that the reaction centers, and the donor and acceptor sides of PSII were all severely damaged after Pst infection. The PSI activity decreased as the infection progressed. Furthermore, we observed a considerable overall degradation of PsbO, D1, PsaA proteins and an over-accumulation of reactive oxygen species (ROS).

Conclusions: Photoinhibition and photoinhibition-like damage were observed under light and dark conditions, respectively, after Pst infection of tobacco leaves. The damage was greater in the dark. ROS over-accumulation was not the primary cause of the photoinhibition and photoinhibition-like damage. The PsbO, D1 and PsaA proteins appear to be the targets during Pst infection under light and dark conditions.
\end{abstract}

Keywords: Biotic stress, Pseudomonas syringae pv. tabaci, Photosystem I, Photosystem II, Nicotiana tabacum

\section{Background}

Under natural conditions, in addition to abiotic stresses, plants are exposed to various biotic stresses, including infection by pathogens and attack by herbivorous pests [1, $2]$. Biotic stresses decrease crop yields worldwide by an average of $15 \%$ [3]. Compared with the number of studies on plant infections caused by fungi and viruses, there are relatively few regarding plants infected by bacteria [4]. The effects of bacterial pathogens infection depends on the severity and timing of infection, but also on the

\footnotetext{
* Correspondence: sungy@vip.sina.com; Fred.Chow@anu.edu.au ${ }^{\dagger}$ Equal contributors

${ }^{1}$ College of Life Science, Northeast Forestry University, Harbin 150040, China Full list of author information is available at the end of the article
}

particular type of bacteria and on genotype-associated host resistance $[5,6]$. Bacterial infections strongly affect photosynthesis. In fact, it has been reported that the genes encoding photosynthetic functions are down regulated [7-9] and changes to photosystem II (PSII) proteins occur in Pseudomonas syringae-infected plants [10].

Pseudomonas syringae are opportunistic bacterial pathogens that can attack a wide variety of plants [11]. There are at least 50 P. syringae pathovars based on their host plant specificities and type of disease symptoms [12, 13]. Previous research has revealed that the maximum PSII quantum yield $\left(F_{\mathrm{v}} / F_{\mathrm{m}}\right)$, the quantum yield of open PSII traps $\left(F_{\mathrm{v}}{ }^{\prime} /\right.$ $\left.F_{\mathrm{m}}{ }^{\prime}\right)$, and nonphotochemical quenching $(\mathrm{NPQ})$ were decreased in Arabidopsis thaliana leaves infected with $P$. 
syringae pv. tomato DC3000 (Pto) [14, 15]. Decreases in the actual photochemical efficiency of PSII $\left(\Phi_{\mathrm{PSII}}\right)$ and NPQ were also observed in Pto-infected Phaseolus vulgaris leaves [16]. Additionally, a decrease in NPQ was observed in $P$. syringae pv. Phaseolicola (Pph)-infected bean plants, while the $F_{\mathrm{v}} / F_{\mathrm{m}}$ remained stable [17]. Moreover, decreases in $\Phi_{\text {PSII }}$ and NPQ were detected in Pph-infected 'Canadian Wonder' $P$. vulgaris leaves [16]. In contrast, a decrease in $F_{\mathrm{v}}{ }^{\prime} / F_{\mathrm{m}}{ }^{\prime}$ and an increase in NPQ were observed in soybean leaves infiltrated with $P$. syringae pv. glycinea [8]. As one of the most important pathovars, $P$. syringae pv. tabaci $(P s t)$ is a hemibiotrophic bacterial pathogen that parasitizes tobacco leaves, causing the formation of brown spots during an infection referred to as wildfire disease $[18,19]$. To better understand how to manage $P$. syringae infections, we focused on the tobacco-Pst model pathosystem. Although considerable research has recently been completed on the tolerance to Pst [20-22] and the photosynthetic performance of plants infected by the other pathovars mentioned above, little information is available on the photosynthetic performance during tobacco-Pst interactions.

The $\mathrm{D}_{1}$ protein is the core protein of the PSII reaction center. The inhibition of photosynthesis electron transport (PET) from the primary quinone electron acceptor of PSII $\left(Q_{\mathrm{A}}\right)$ to the secondary quinone electron acceptor of PSII $\left(Q_{\mathrm{B}}\right)$ may consequently be related to the degradation of the $\mathrm{D}_{1}$ protein [23]. Similarly, PsbO, the core component of the oxygen evolving complex (OEC), is critical to the functionality of the OEC [24]. Additionally, photosystem I (PSI) photoinhibition is related to the degradation of PsaA [25]. In several studies, dark conditions were simulated using the PET inhibitors 3(3,4-dichlorophenyl)-1,1-dimethylurea and 2,5-dibromo3-methyl-6-isopropylbenzoquinone [26, 27]. However, this study focused on PET as influenced by Pst infection. Therefore, these inhibitors were not used.

Our objectives were to identify the differences in PSI and PSII responses to light and dark conditions following Pst infection of tobacco leaves. We also aimed to determine if photoinhibition occurs during Pst infection. To address these questions, we (1) evaluated the changes to the donor and acceptor sides and the reaction center of PSII as well as the PSI activity after Pst infection, (2) monitored the production of reactive oxygen species (ROS), and (3) performed Western blot analyses of the thylakoid membrane proteins of treated tobacco leaves. We compared the responses of the photosynthetic apparatus to Pst infection under light and dark conditions.

\section{Results}

Effects of Pst infection on chlorophyll content in the infiltrated area of tobacco leaves

We observed chlorotic lesions in the infiltrated zone at 3 days post infection (dpi), while necrosis was observed at 3 dpi only in leaves treated in the dark. The infiltrated zone of tobacco leaves exhibited obvious wildfire symptoms regardless of whether the leaves were incubated under light or dark conditions (Fig. 1). The total chlorophyll content in infected leaves at 3 dpi was lower than that of untreated leaves (Fig. 2).

\section{Effects of Pst infection on the donor and acceptor sides and the reaction center of PSII in tobacco leaves}

We used the JIP-test to detect PSII changes in Pst-infected tobacco leaves under light and dark conditions. To clarify the effects of Pst on PSII, OJIP curves were normalized to the $\left(F_{\mathrm{m}}-F_{\mathrm{o}}\right)$ level. The shape of the OJIP transient changed over time, with the $\mathrm{K}$ and J points increasing markedly and the amplitude increasing along with the inoculation time (Fig. 3). The K step (at $300 \mu$ s) of the chlorophyll $a$ fluorescence transient (quantified as $W_{\mathrm{K}}$ ) has been widely used as a specific indicator of oxygen evolving complex (OEC) injury in the photosynthetic apparatus [28, 29]. We observed that $W_{\mathrm{K}}$ increased after Pst infection under light and dark conditions. The increase was more pronounced with increasing time, suggesting that the activity of the donor side of PSII was inhibited and that the OEC was damaged. Compared with that of untreated leaves, $W_{\mathrm{k}}$ increased by 12.9 and $25.6 \%$ at 3 dpi under light and dark conditions, respectively (Fig. 4a, b). The relative variable fluorescence at the J-step $\left(V_{\mathrm{J}}\right)$ represents the subsequent kinetic bottleneck of the electron transport chain, resulting in the momentary maximum accumulation of $Q_{\mathrm{A}}^{-}[30,31] . V_{\mathrm{J}}$ is an indicator of the level of closure of PSII reaction centers or the redox state of $Q_{\mathrm{A}}$ [32]. In this study, compared with untreated leaves, $V_{\mathrm{J}}$ increased by 13.9 and $103 \%$ in the infiltrated zone at $3 \mathrm{dpi}$ under light and dark conditions, respectively (Fig. 4c, d). Thus, electron transport from $Q_{\mathrm{A}}$ to $Q_{\mathrm{B}}$ was severely blocked after Pst infection in tobacco leaves. Moreover, inhibition of the $\mathrm{K}$ and $\mathrm{J}$ steps was more pronounced in the dark, as indicated by the greater increase of the $W_{\mathrm{k}}$ and $V_{\mathrm{J}}$ values in the dark during Pst inoculation (Fig. 4a-d). The maximum quantum yield of PSII $\left(F_{\mathrm{v}} /\right.$ $\left.F_{\mathrm{m}}\right)$ and the density of $Q_{\mathrm{A}}^{-}$reducing PSII reaction centers per cross section $(\mathrm{RC} / \mathrm{CSm})$ values decreased to 94.7 nd $85.4 \%$ of the values of untreated leaves (under light conditions) at $3 \mathrm{dpi}$, respectively (Fig. $4 \mathrm{e}, \mathrm{g}$ ). The $F_{\mathrm{v}} /$ $F_{\mathrm{m}}$ and $\mathrm{RC} / \mathrm{CSm}$ values of treated leaves decreased to 91.9 and $66.8 \%$ of the values of untreated leaves (under dark conditions) at $3 \mathrm{dpi}$, respectively (Fig. $4 \mathrm{f}, \mathrm{h}$ ).

\section{Effects of Pst infection on PSI complex activity in tobacco leaves}

We observed considerable differences in PSI activity among treated leaves. The PSI complex activities of treated leaves were 80.0 and $70.8 \%$ of the activity of untreated leaves at 3 dpi under light and dark conditions, 

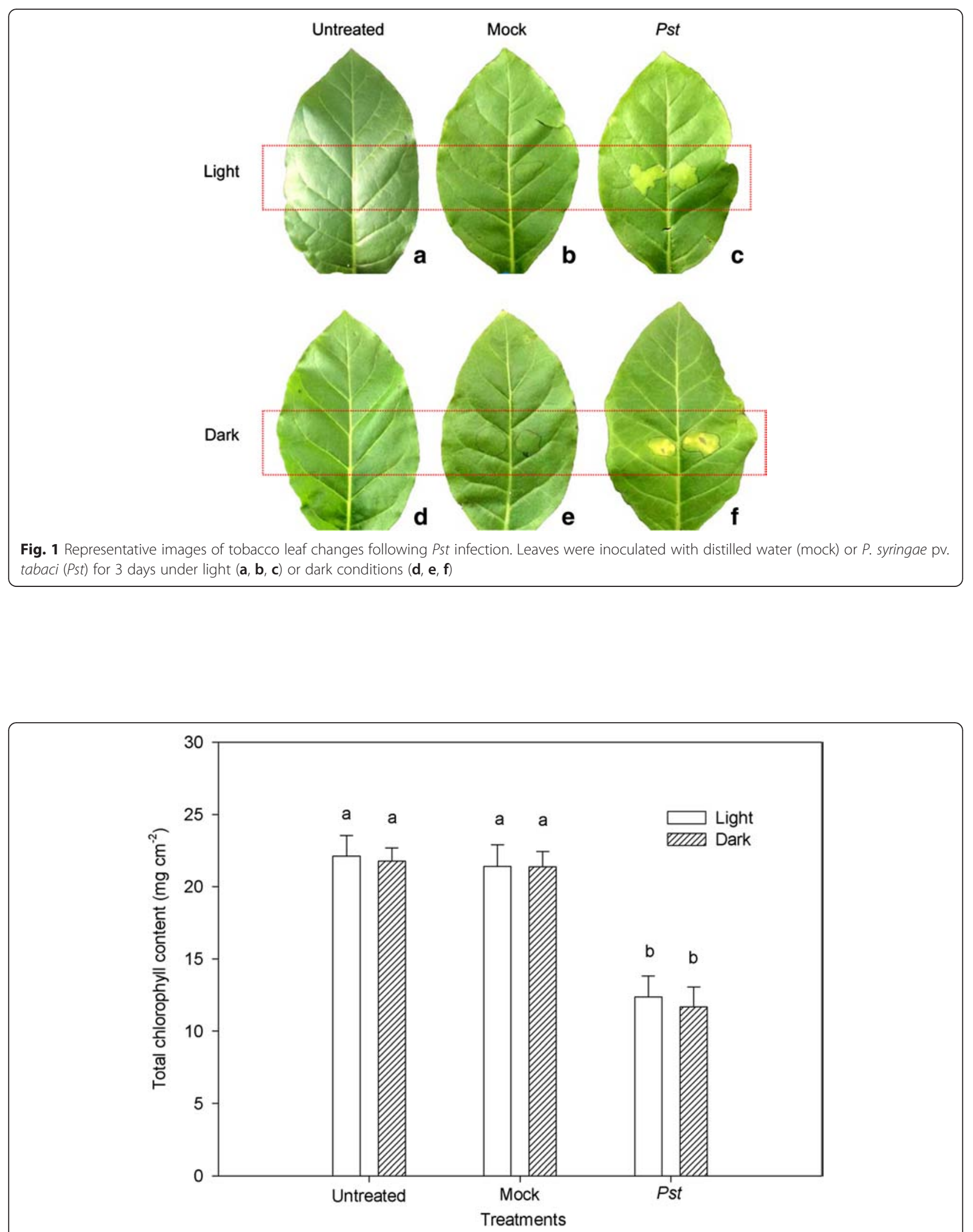

Fig. 2 Relative changes in total chlorophyll content at 3 days post Pst infection in tobacco leaves. Means \pm SE of three replicates are presented. Different letters above the columns indicate significant differences at $P<0.05$ between different treatments 


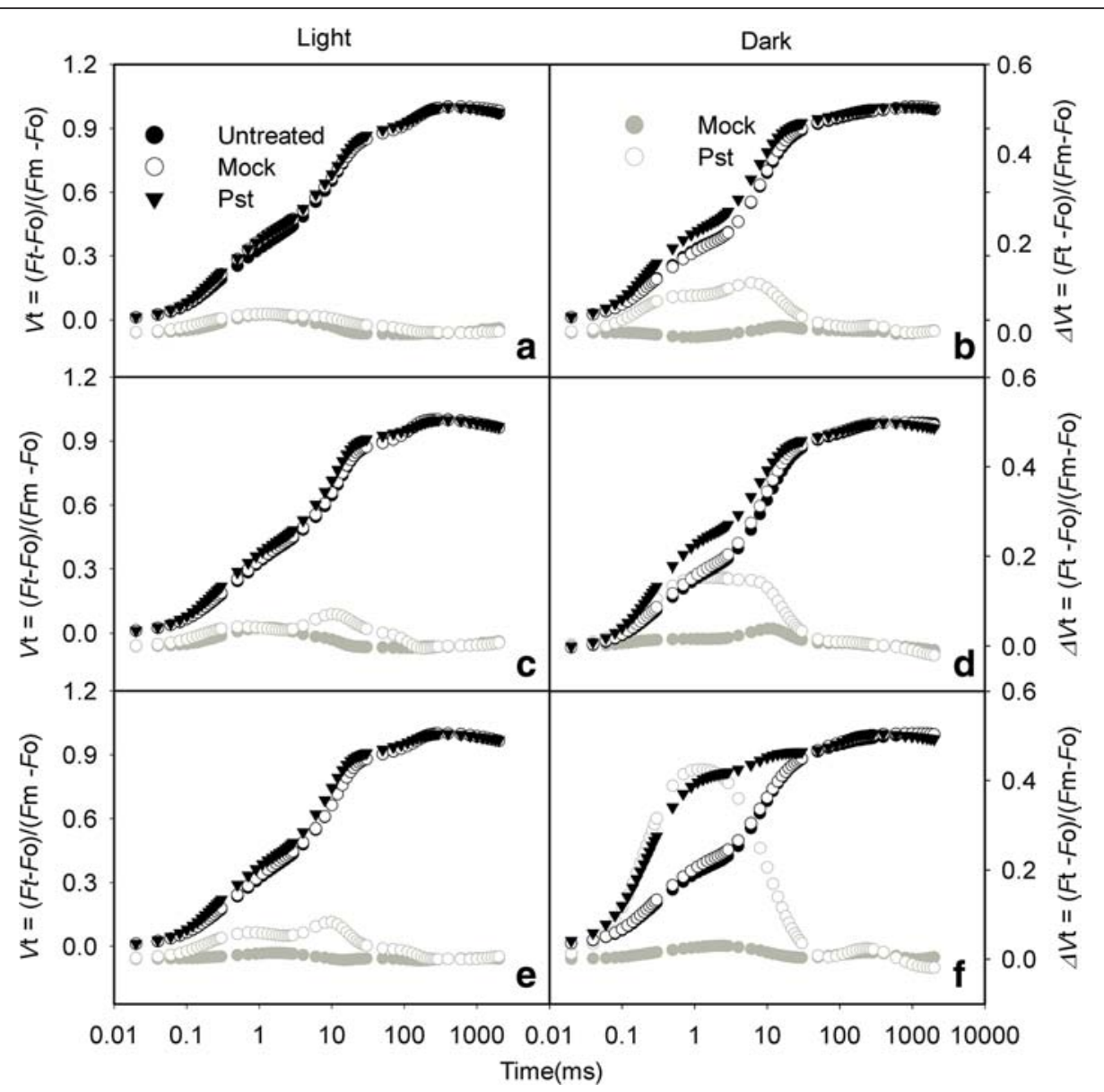

Fig. 3 Relative changes in chlorophyll fluorescence induction kinetics during Pst inoculation of tobacco leaves. Leaves were inoculated with distilled water (mock) or P. syringae pv. tabaci (Pst) for $1(\mathbf{a}, \mathbf{b}), 2$ days $(\mathbf{c}, \mathbf{d})$, or 3 days $(\mathbf{e}, \mathbf{f})$ under light or dark conditions. The $K$ point indicates the $\mathrm{K}$ step at about $300 \mu$ s and the J point indicates the J step at about $2 \mathrm{~ms}$. $\Delta V_{\mathrm{t}}$ was determined by subtracting the kinetics of the untreated leaves from the kinetics of leaves treated with distilled water or Pst. The black symbols correspond to the left y axis and the grey symbols correspond to the right y axis. Every curve is the average of 10 replicates

respectively (Fig. 5). This indicates that P700 photooxidation was rapidly and effectively impaired by Pst infection in tobacco leaves under light and dark conditions. Further, the extent of the decrease in PSI activity was greater in the dark (Fig. 5).

\section{Effects of Pst infection on carbon assimilation in tobacco leaves}

The net photosynthetic rate $(\mathrm{Pn})$, stomatal conductance (Gs), and carboxylation efficiency (CE) values of treated leaves were 69.3, 17.5, and $21.1 \%$ lower than those of mock controls at $3 \mathrm{dpi}$, respectively. In contrast, the intercellular $\mathrm{CO}_{2}$ concentration $(\mathrm{Ci})$ value of treated leaves was $23.6 \%$ higher than that of mock controls at 3 dpi (Table. 1).

\section{Relative ROS level changes after Pst infection in tobacco leaves}

We evaluated $\mathrm{H}_{2} \mathrm{O}_{2}$ production in the Pst-infiltrated zone of tobacco leaves at 3 dpi under light and dark conditions because $\mathrm{H}_{2} \mathrm{O}_{2}$ is the most stable ROS that can be readily measured [33]. The production of $\mathrm{H}_{2} \mathrm{O}_{2}$ was evaluated in the Pst-infiltrated zone of tobacco leaves at $3 \mathrm{dpi}$ under light and dark conditions. The $\mathrm{H}_{2} \mathrm{O}_{2}$ content of treated leaves were 269 and $112 \%$ higher than that of untreated controls at 3 dpi under light and dark conditions, respectively (Fig. 6). This implies that an over-accumulation of ROS was induced by Pst infection in tobacco leaves under light and, to a lesser extent, dark conditions.

\section{Pst-induced degradation of PsbO, D1, and PsaA proteins in tobacco leaves}

The D1 protein pool sizes is representative of the abundance of fully assembled PSII centers as there is one D1 subunit per reaction center. The mature protein is thought to accumulate only when it is integrated into PSII reaction centers. The content of PsbO, D1, and PsaA proteins decreased to 67.0, 65.1 and $70.0 \%$ of the values of water-treated leaves at $3 \mathrm{dpi}$ under light 


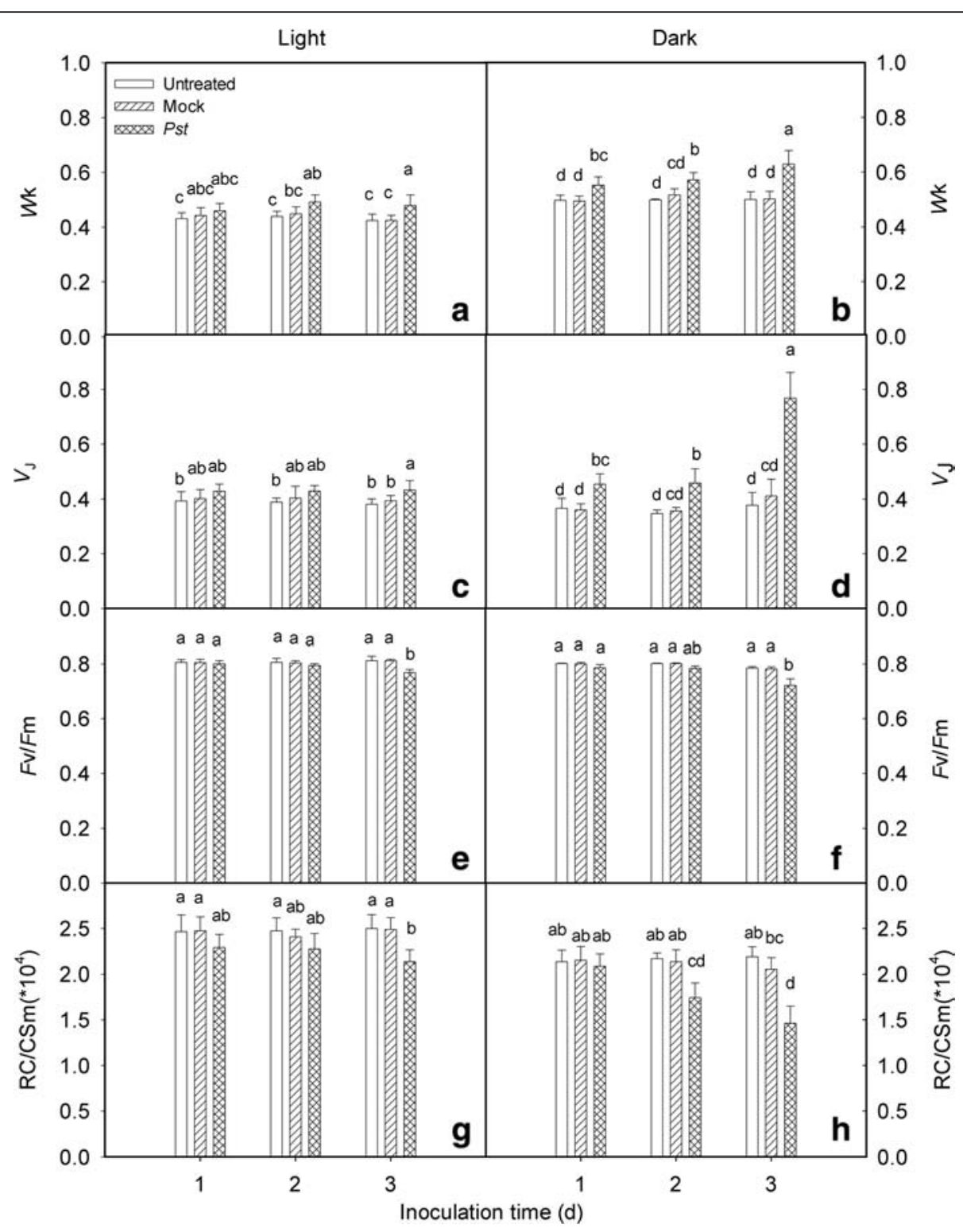

Fig. 4 Relative changes in $W_{\mathrm{K}}, V_{\mathrm{J}}, F_{\mathrm{v}} / F_{\mathrm{m}}$, and RC/CSm after Pst infection in tobacco leaves. Chlorophyll a fluorescence transients were analyzed with the JIP-test. The $W_{K}(a, b), V_{J}(c, d), F_{v} / F_{m}(e, f)$, and RC/CSm $(g, h)$ values were calculated after tobacco leaves were inoculated with distilled water (mock) or $P$. syringae pv. tabaci (Pst) for specific periods under light or dark conditions. Means \pm SE of 10 replicates are presented. Different letters above the columns indicate significant differences at $P<0.05$ between different treatments

conditions, respectively. The core proteins decreased to $44.1,51.0$ and $50.2 \%$ of the values of water-treated leaves at 3 dpi under dark conditions, respectively (Fig. 7).

\section{Discussion}

We observed lesions consisting of a necrotic center surrounded by chlorotic tissue at 3 dpi in the dark (Fig. 1). Plant pathogens can generally be categorized in three classes (necrotrophs, biotrophs, and hemibiotrophs) on the basis of mechanisms of infection. Biotrophics need living tissue for growth and reproduction. Necrotrophics kill the host tissue during the initial stages of infection and feed on the dead tissue. Hemi-biotrophics exist as biotrophs before switching to a necrotrophic stage [34].

Our study revealed that chlorophyll content decreased considerably during Pst inoculation under light and dark conditions (Fig. 2). Chlorophyll degradation has been observed in several plant - pathogen interactions $[35,36]$. Kudoh and Sonoike reported that in the early recovery stage after PSI damage, chlorophyll degradation occurred to prevent the absorption of excessive light energy which can otherwise lead to secondary injury of the photosystems [37]. Moreover, Thomas reported that tabtoxinine- $\beta$-lactam, a toxin originally 

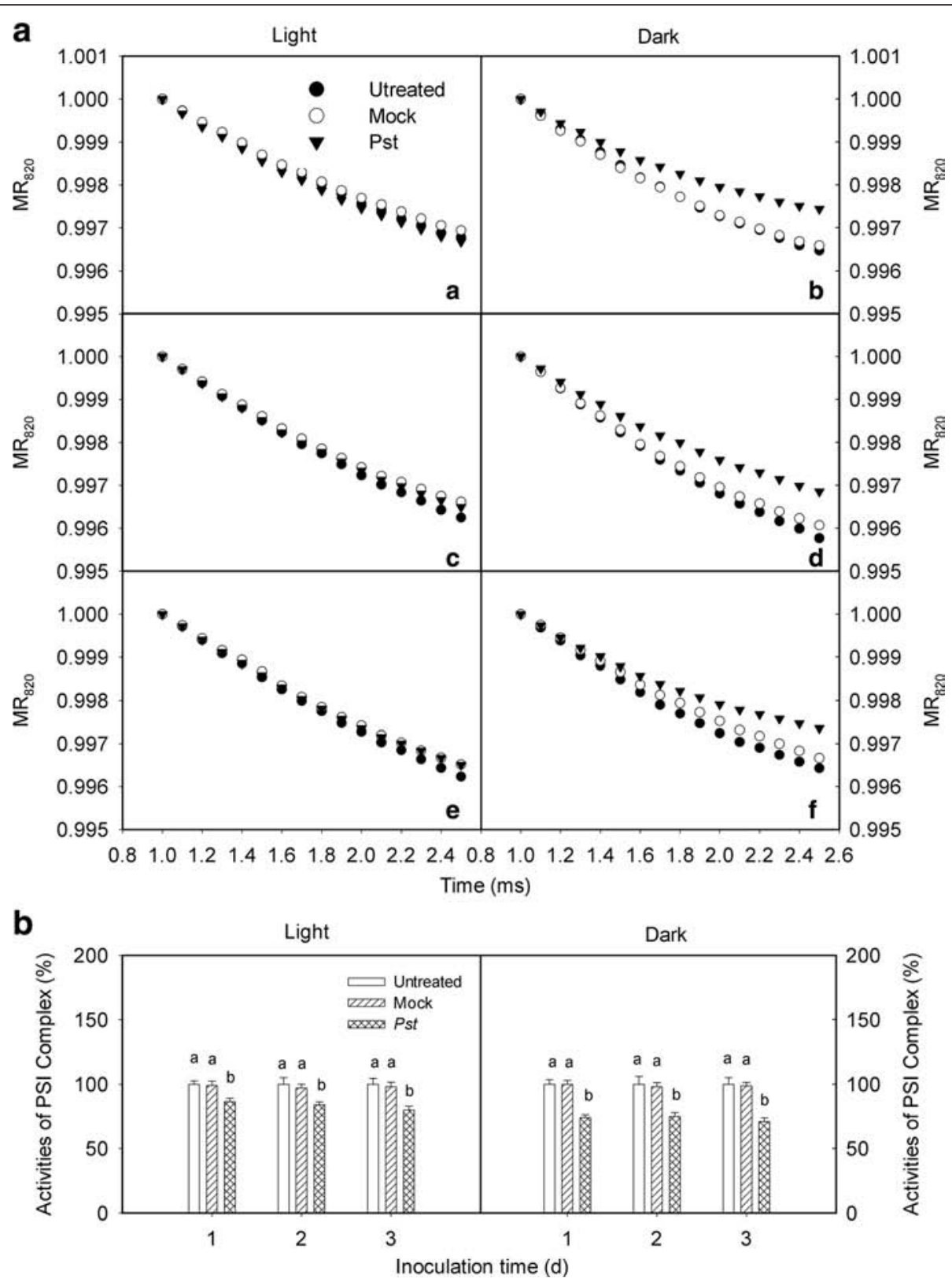

Fig. 5 Relative changes in PSI complex activity after Pst infection in tobacco leaves. a. Modulated reflected signal of $820 \mathrm{~nm}\left(\mathrm{MR}_{820 \mathrm{~nm}}\right)$ was evaluated after leaves had been inoculated with distilled water (mock) or P. syringae pv. tabaci (Pst) for 1 ( $a, b), 2$ (c, d), or 3 days (e, f) under light and dark conditions. The treated leaves were illuminated with red light $(2.5 \mathrm{~s})$ and the MR820nm signal changes were simultaneously recorded. The initial $M_{820 n m}$ rate indicates PSI activity. Every curve is the average of 10 replicates. $\mathbf{b}$. The PSI complex activity was evaluated after leaves were inoculated with distilled water (mock) or Pst for different periods under light (a) and dark (b) conditions. The initial PSI complex activity of untreated tobacco leaves was considered $100 \%$, while the activities of mock- and Pst-treated leaves were calculated as the percentage of activity in untreated leaves. Means \pm SE of 10 replicates are presented. Different letters above the columns indicate significant differences at $P<0.05$ between different treatments

described as being from Pst, is a dipeptide whose hydrolysis product irreversibly inhibits glutamine synthetase and induces chlorophyll degradation in tobacco leaves [38]. Therefore, the putative tabtoxin activity of Pst and the need for photoprotection of the tobacco leaves after PSI damage may have been responsible for the observed chlorophyll degradation.
The reduction of $\mathrm{Pn}$ in leaves may have been due to limited $\mathrm{CO}_{2}$ diffusion to carboxylation sites as a consequence of decreased stomatal conductance or because of perturbation of enzymatic processes in the Calvin cycle [39]. The decreased Gs and the increased $\mathrm{Ci}$ in the Pst infiltrated leaves (Table 1) indicated that the decrease in Pn may be the result of a non-stomatal limitation. The 
Table 1 Relative changes to carbon assimilation parameters at 3 days post Pst infection in tobacco leaves

\begin{tabular}{lcccc}
\hline & $\mathrm{Pn}\left(\mu \mathrm{mol} \mathrm{m} \mathrm{s}^{-1}\right)$ & $\mathrm{Gs}\left(\mathrm{mmol} \mathrm{m}^{-2} \mathrm{~s}^{-1}\right)$ & $\left.\mathrm{Ci}(\mu \mathrm{mol} \mathrm{mol})^{-1}\right)$ & $\mathrm{CE}\left(\mu \mathrm{mol} \mathrm{m}^{-2} \mathrm{~s}^{-1}\right)$ \\
\hline Mock & $5.8 \pm 0.53 \mathrm{a}$ & $63 \pm 5.29 \mathrm{a}$ & $225 \pm 16.5 \mathrm{~b}$ & $0.0521 \pm 0.006 \mathrm{a}$ \\
Pst & $1.78 \pm 0.23 \mathrm{~b}$ & $52 \pm 6.08 \mathrm{~b}$ & $278 \pm 20.6 \mathrm{a}$ & $0.0411 \pm 0.008 \mathrm{~b}$ \\
\hline
\end{tabular}

The changes to net photosynthetic rate (Pn), stomatal conductance (Gs), intercellular $\mathrm{CO}_{2}$ concentration (Ci), and carboxylation efficiency (CE) were evaluated. The mean \pm SE of four replicates are shown. Different small letters present on the same column indicate significant differences at $P<0.05$ between different treatments

decrease in CE (Table 1) indicates that the ribulose 1, 5bisphosphate carboxylase/oxygenase activity may be inhibited by Pst infection, leading to the inhibition of $\mathrm{CO}_{2}$ assimilation. Photosynthetic electron transport and carboxylation were both inhibited by Pst infection. However, it is unclear whether the effects on PET are the result of inhibition of downstream carboxylation.

The phosphoenolpyruvate carboxylase (EC 4.1.1.31, PEPc) catalyses the irreversible $\beta$-carboxylation of phosphoenolpyruvate using $\mathrm{HCO}_{3}^{-}$as a substrate in a reaction that yields oxaloacetic acid and inorganic phosphate [40]. Several papers have shown that PEPc activity increased in salt treated Sorghum bicolor (a C4 plant), Hordeum vulgare (a C3 plant) and Aleuropus litoralis (a C3-C4 intermediate plant) [41-43]. The activity of PEPc increased after Potato virus $Y$ or Potato virus $A$ infection in tobacco leaves $[44,45]$. This stimulation of PEPc activity under biotic and abiotic stresses would allow replenishment of the tricarboxylic acid cycle to maintain the activated internal nitrogen metabolism in spite of the reduced photosynthesis rate [46].

The decreases in $F_{\mathrm{v}} / F_{\mathrm{m}}$ and $\mathrm{RC} / \mathrm{CSm}$ are conventional indicators of photoinhibition under light conditions [47]. The $F_{\mathrm{v}} / F_{\mathrm{m}}$ and $\mathrm{RC} / \mathrm{CSm}$ values decreased considerably as the Pst infection progressed (Fig. 4), suggesting that
Pst infection causes photoinhibition of PSII under light conditions.

Photosystem II is considered to be more vulnerable than PSI when plants encounter stresses because few species have been found in which PSI is more easily photoinhibited than PSII $[48,49]$. Photoinhibition of PSI was first reported by Terashima et al. in cucumber plants exposed to low temperature [50]. The PSI activity decreased after Pst infection (Fig. 5), indicating that PSI photoinhibition occurred during Pst inoculation under light conditions. However, we observed damages to the photosynthetic apparatus during Pst inoculation under dark conditions that were similar to the damage caused by photoinhibition induced by light. Therefore, this damage was referred to as "photoinhibition-like damage" which was further indicated by the degradation of PsbO, D1, and PsaA proteins (Fig. 7).

Chloroplasts are the major source of ROS in plant cells. The direct reduction of $\mathrm{O}_{2}$ to superoxide by reduced donors associated with PSI occurs during the Mehler reaction [51]. The impairment of photosystems inevitably leads to the generation of ROS by the Mehler reaction during Pst inoculation (Fig. 6). There are two roles for $\mathrm{H}_{2} \mathrm{O}_{2}$ in plants. At low concentrations, it acts as a messenger molecule involved in signaling related to

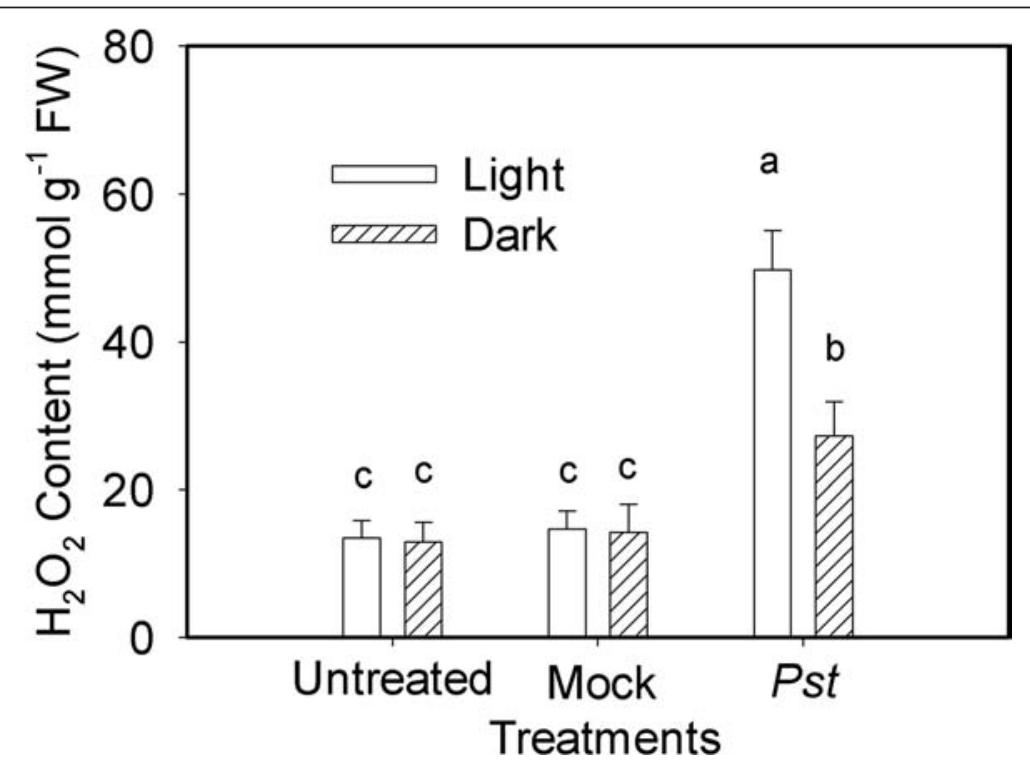

Fig. 6 Relative changes in $\mathrm{H}_{2} \mathrm{O}_{2}$ content at 3 days post Pst infection in tobacco leaves. Means \pm SE of 10 replicates are presented. Different letters above the columns indicate significant differences at $P<0.05$ between different treatments 


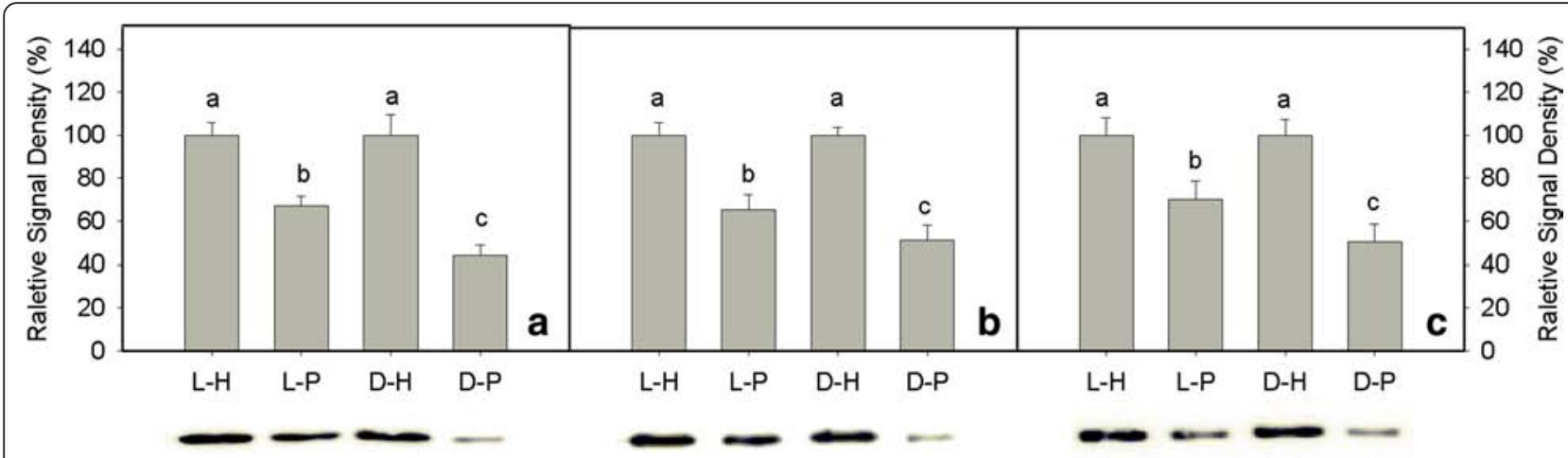

Fig. 7 Quantitative image analysis of core protein levels at 3 days post infection in tobacco leaves. PsbO (a), D1 (b), and PsaA (c) protein levels were evaluated. L-H represents leaves infiltrated with distilled water in the light; L-P represents leaves infiltrated with $P$. syringae pv. tabaci (Pst) in the light; D-H represents leaves infiltrated with distilled water in the dark; and D-P represents leaves infiltrated with Pst in the dark. For complete Western blots of PsbO, D1, and PsaA, please see Additional file 1, Additional file 2, Additional file 3. The relative signal density of mock controls was considered $100 \%$, while the signal density of Pst treatments were calculated as the percentage of density in mock controls. Means \pm SE of three replicates are presented. Different letters above the columns indicate significant differences at $P<0.05$ between different treatments

acclimation and the triggering of defense mechanisms against various stresses [52]. At high concentrations, $\mathrm{H}_{2} \mathrm{O}_{2}$ promotes programmed cell death and oxidative damage [53]. Additionally, $\mathrm{H}_{2} \mathrm{O}_{2}$ can suppress de novo D1 protein synthesis by inhibiting elongation factor $G$ $[54,55]$. Several reports have suggested that ROS overproduction is involved in photoinhibition during various stresses [56, 57]. However, the observed damage to the photosystems was greater and the increase in $\mathrm{H}_{2} \mathrm{O}_{2}$ was much smaller in the dark than in the light (Fig. 6). These results suggest that ROS over-accumulation was not the main reason for the photoinhibition and photoinhibition-like damage induced by Pst in tobacco leaves. Additionally, PSI is likely to be attacked by ROS during exposure to stresses, but this attack occurs only if the reduced state of iron-sulfur centers can be maintained, which requires visible light [58]. However, the damage to PSI was greater in the dark, further supporting the viewpoint mentioned above. In accordance with this, Fan et al. indicated that the photoinhibition-like damage of daylily, willow, euonymus japonicus and maize was not caused by the over-accumulation of ROS under dark conditions [59].

Counteracting to the negative effects of ROS on the photosynthetic apparatus during photoinhibition, the greater abundance of $\mathrm{H}_{2} \mathrm{O}_{2}$ under light conditions may have led to increased hydroxyl free radical production by the Fenton reaction. The hydroxyl radical may inhibit the pathogen under light conditions [60]. This may be a positive effect of $\mathrm{H}_{2} \mathrm{O}_{2}$ that helped to alleviate photoinhibition and photoinhibition-like damage.

The production of ATP and NADPH during photosynthesis decreases in the dark [61]. The replacement of damaged PSII proteins (primarily the D1 protein) with newly synthesized proteins is an ATP-dependent process [62]. Additionally, the synthesis of the D1 protein of the PSII heterodimer, which is the most rapidly synthesized chloroplast protein, is stimulated by bright light [63] Therefore, the limited recovery of PSII under dark conditions may be one of the reasons for the greater overall damage observed in the dark during Pst inoculation. If a partially repaired PSII in the light minimized the overall damage to the photosystem, it is unclear why the damage to PSI was less extensive in the light than in the dark. The repair of PSI is a very slow process that requires several days or longer. Therefore, the results can not be related to PSI repair. Further studies are needed to clarify this point.

\section{Conclusions}

We evaluated the response of PSI and PSII to Pst infection in tobacco leaves under light and dark conditions. The reaction centers and the donor and acceptor sides of the photosystems were all severely damaged, indicating that photoinhibition and photoinhibition-like damage had occurred. We also observed a considerable (net) degradation of PsbO, D1, and PsaA proteins and an over-accumulation of ROS. The accumulated ROS, however, was not the main reason for the photoinhibition and photoinhibition-like damage induced by Pst in tobacco leaves. The PsbO, D1, and PsaA proteins appear to be the targets of Pst infection under light and dark conditions. Further investigations of photosystem responses may help to identify the main sites of Pst-induced damaged in tobacco leaves. This will lead to a better understanding of the mechanisms of plantpathogen interactions and assist in the breeding of Psttolerant species. 


\section{Methods}

Plant materials and infiltration with Pst

Seeds of tobacco (Nicotiana tabacum cv. Longjiang 911, a susceptible cultivar, was kindly supplied by Dr. JianPing Sun, Tobacco Research Institute of Mudanjiang, Mudanjiang, China) were germinated on vermiculite. Forty-five days after germination, the seedlings were transplanted to pots containing a compost-soil substrate to grow in a greenhouse under a natural photoperiod. The two upper fully expanded attached leaves of six to eight weeks old plants were used for experiments.

Pseudomonas syringae pv. tabaci were grown on solid King's B agar plates overnight [64], diluted with distilled water to a concentration $10^{6}$ colony forming units per milliliter. Distilled water (mock) or bacterial suspensions were hand-infiltrated into mesophyll with a needleless syringe on the abaxial side of the leaves. Infiltrating area was about $1 \mathrm{~cm}^{-2}$ and measurements were made at a distance of about $0.5 \mathrm{~cm}$ from the infiltration area. Following inoculation, the leaves were kept under $14 \mathrm{~h}$ light $\left(200 \mu \mathrm{mol} \mathrm{m} \mathrm{m}^{-2} \mathrm{~s}^{-1}\right) / 10 \mathrm{~h}$ dark cycles or continuous darkness at $25^{\circ} \mathrm{C}$.

\section{Measurements of total chlorophyll content in tobacco leaves after Pst infection}

Leaf total chlorophyll was extracted with $80 \%$ acetone in the dark for $72 \mathrm{~h}$ at $4{ }^{\circ} \mathrm{C}$. The extracts were analyzed using a UV-visible spectrophotometer UV1601 (Shimadzu, Japan) according to the method of Porra (2002) [65].

\section{Measurement of gas exchange in tobacco leaves after Pst infection}

The Pn, Gs, and Ci were measured by a CIRAS-3 portable photosynthetic system (PP Systems, USA), which controls the photosynthetic photon flux density at $800 \mu \mathrm{mol} \mathrm{m}{ }^{-2} \mathrm{~s}^{-1}$, temperature at $25{ }^{\circ} \mathrm{C}$ and $\mathrm{CO}_{2}$ concentration at $390 \mu \mathrm{mol} \mathrm{mol}{ }^{-1}$ in the leaf chamber. $\mathrm{CO}_{2}$ concentration was changed every $3 \mathrm{~min}$ in a sequence of 1 600, 1 200, 800, 600, 400, 300, 200, 150, 100 and $0 \mu \mathrm{mol} \mathrm{mol}{ }^{-1}$. Irradiance and $\mathrm{CO}_{2}$ concentration were controlled by the automatic control function of the system. CE was calculated according the initial slop of Pn$\mathrm{Ci}$ response curve [66].

Measurements of the chlorophyll $a$ fluorescence transient (OJIP) and PSI activity in tobacco leaves after Pst infection Induction kinetics of prompt fluorescence and the modulated reflected signal of $820 \mathrm{~nm}\left(M_{820} \mathrm{~nm}\right)$ were simultaneously recorded using a Multifunctional Plant Efficiency Analyzer, M-PEA (Hansatech Instrument Ltd., UK) as has been described [67]. All leaves were dark adapted before measurements. Chlorophyll a fluorescence transients were analyzed with the JIP- test: $F_{\mathrm{v}} / F_{\mathrm{m}}=1-\left(F_{\mathrm{o}} / F_{\mathrm{m}}\right) ; V_{\mathrm{J}}=\left(\mathrm{F}_{2} \mathrm{~ms}-\mathrm{F}_{\mathrm{o}}\right) /\left(\mathrm{F}_{\mathrm{m}}-\mathrm{F}_{\mathrm{o}}\right)$; $W_{\mathrm{k}}=\left(F_{0.3 \mathrm{~ms}}-F_{\mathrm{o}}\right) /\left(F_{2 \mathrm{~ms}}-F_{\mathrm{o}}\right) ; \mathrm{RC} / \mathrm{CSm}=\phi_{\text {Po }} \cdot\left(V_{\mathrm{J}} /\right.$ $\left.M_{\mathrm{o}}\right) \cdot(\mathrm{ABS} / \mathrm{CSm})$, and $\mathrm{M}_{\mathrm{o}}=4\left(F_{0.3 \mathrm{~ms}}-F_{\mathrm{o}}\right) /\left(F_{\mathrm{m}}-\right.$ $\left.F_{\mathrm{o}}\right) ; \phi_{\mathrm{Po}}=F_{\mathrm{v}} / F_{\mathrm{m}}$. The $\mathrm{MR}_{820} \mathrm{~nm}$ signal measured at $820 \mathrm{~nm}$ provides information about oxidation state of PSI, including plastocyanin and P700. The induction curve of $M_{820} \mathrm{~nm}$ of the leaves obtained by saturating red light showed a fast oxidation phase and a subsequent reduction phase. The initial slope of the oxidation phase of $\mathrm{MR}_{820 \mathrm{~nm}}$ at the beginning of the saturated red light indicates the capability of P700 to get oxidized, which is used to reflect the activity of PSI $[68,69]$.

\section{Detection of $\mathrm{H}_{2} \mathrm{O}_{2}$ generation in tobacco leaves after Pst infection}

$\mathrm{H}_{2} \mathrm{O}_{2}$ was extracted and determined according to the method of Patterson [70]. Leaf segments $(0.5 \mathrm{~g})$ were ground in liquid nitrogen, extracted with $5 \mathrm{ml}$ of $5 \%$ $(\mathrm{w} / \mathrm{v})$ trichloroacetic acid and then centrifuged at 16 $000 \times g$ for $10 \mathrm{~min}$. The supernatant was used for the $\mathrm{H}_{2} \mathrm{O}_{2}$ assay.

\section{Detection of Psb O, D1, and PsaA proteins in tobacco leaves after Pst infection}

Thylakoid membranes proteins were detected by Western blot with equal amounts of chlorophyll. Leaves were homogenized in an ice cold isolation buffer [100 mM sucrose, $50 \mathrm{mM}$ Hepes (pH 7.8), $20 \mathrm{mM} \mathrm{NaCl}, 2 \mathrm{mM}$ EDTA and $2 \mathrm{mM} \mathrm{MgCl}_{2}$ ], then filtered through three layers of pledget. The filtrate was centrifuged at $3000 \times g$ for $10 \mathrm{~min}$. The sediments were washed with isolation buffer, re-centrifuged, and then finally suspended in an isolation buffer. The thylakoid membrane proteins were then denatured and separated using $12 \%$ polyacrylamide gradient gel. The denatured proteins in the gel were then electro-blotted to PVDF membranes, probed with antibodies supplied by Fan et al. [59] and then visualized by a chemiluminescence method. Quantitative image analysis of protein levels was performed with Gel-Pro Analyzer 4.0 software.

\section{Chemicals used in the study}

All the compounds used in this study were manufactured by Sigma.

\section{Statistical analysis}

The results presented were the means of at least three independent measurements. Means were compared by analysis of variance and LSD range test at $5 \%$ level of significance.

\section{Availability of data and materials}

All the supporting data are included as additional files. 


\section{Additional files}

Additional file 1: Figure S1. PsbO protein level was evaluated at 3 days post infection in tobacco leaves. Lanes from left to right in the picture represent leaves infiltrated with distilled water in the light, leaves infiltrated with $P$. syringae pv. tabaci (Pst) in the light, leaves infiltrated with distilled water in the dark, and leaves infiltrated with Pst in the dark, respectively (PNG $9 \mathrm{~kb}$ )

Additional file 2: Figure S2. D1 protein level was evaluated at 3 days post infection in tobacco leaves. Lanes from left to right in the picture represent leaves infiltrated with distilled water in the light, leaves infiltrated with $P$. syringae pv. tabaci (Pst) in the light, leaves infiltrated with distilled water in the dark, and leaves infiltrated with Pst in the dark, respectively (PNG $7 \mathrm{~kb}$ )

Additional file 3: Figure S3. PsaA protein level was evaluated at 3 days post infection in tobacco leaves. Lanes from left to right in the picture represent leaves infiltrated with distilled water in the light, leaves infiltrated with P. syringae pv. tabaci (Pst) in the light, leaves infiltrated with distilled water in the dark, and leaves infiltrated with Pst in the dark, respectively (PNG $10 \mathrm{~kb}$ )

\section{Abbreviations}

CE: Carboxylation efficiency; $\mathrm{Ci}$ : Intercellular $\mathrm{CO}_{2}$ concentration; Dpi: Days post infection; $F_{0}: F_{m}$ Initial and maximum fluorescence; $F_{v} / F_{m}$ : Maximal quantum yield of PSII; $F_{\mathrm{v}}{ }^{\prime} / F_{\mathrm{m}}$ : The quantum yield of open PSII traps; Gs: Stomatal conductance; J: K, Intermediate steps of chlorophyll a fluorescence rise between $F_{0}$ and $F_{m} ; R_{820} \mathrm{~nm}$ : Modulated reflected signal of 820 nm; mSR705: The modified red-edge ratio; NPQ: Nonphotochemical quenching; OEC: Oxygen evolving complex; PEPc: Phosphoenolpyruvate carboxylase; PET: Photosynthesis electron transport; Pn: Net photosynthetic rate; Pph: Pseudomonas pv. Phaseolicola; PSI: Photosystem I; PSII: Photosystem II; Pst: Pseudomonas syringae pv. tabaci; Pto: Pseodomonas syringae pv. tomatao DC300; $Q_{A}$ : The primary quinone electron acceptor of PSIl; $Q_{B}$ : The secondary quinone electron acceptor of PSII; RC/CSm: Density of $Q_{A}^{-}$ reducing PSII reaction centre; ROS: Reactive oxygen species; $V_{j}$ : The relative variable fluorescence at the $J$ step; $V_{t}$ : The relative variable fluorescence at the any time; $W_{\mathrm{K}}$ : Normalized relative variable fluorescence at the $\mathrm{K}$ step; $\Phi_{\text {PSII: }}$ The actual photochemical efficiency of PSII.

\section{Competing interests}

The authors declare that they have no competing interests.

\section{Authors' contributions}

DDC, ZSZ, GYS and XBS designed the study. DDC and ZSZ carried out most of the experiments and data analysis. DDC, WSC and MZ conceived of the study, and helped to draft and revise the manuscript. All authors read and approved the final manuscript.

\section{Acknowledgements}

This work was supported by the Fundamental Research Funds for the Central Universities (No. 2572014AA18), China National Nature Science Foundation (No. 31070307) and Outstanding Academic Leaders for Innovation Talents of Science and Technology of Harbin City in Heilongjiang Province (No. 2013RFXXJ063).

\section{Author details}

${ }^{1}$ College of Life Science, Northeast Forestry University, Harbin 150040, China. ${ }^{2}$ State Key Lab of Crop Biology, College of Life Sciences, College of Horticulture Science and Engineering, Shandong Agricultural University, Tai'an 271018, China. ${ }^{3}$ Division of Plant Science, Research School of Biology, College of Medicine, Biology and Environment, The Australian National University, Acton ACT 2601, Australia.

\section{Received: 17 November 2015 Accepted: 21 January 2016}

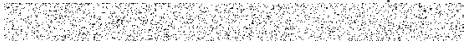

\section{References}

1. Atkinson NJ, Urwin PE. The interaction of plant biotic and abiotic stresses: from genes to the field. J Exp Bot. 2012;63(10):3523-43.
2. Berger S, Sinha AK, Roitsch T. Plant physiology meets phytopathology: plant primary metabolism and plant-pathogen interactions. J Exp Bot. 2007; 58(15-16):4019-26.

3. Oerke EC, Dehne HW. Safeguarding production losses in major crops and the role of crop protection. Crop Prot. 2004;23(4):275-85.

4. Barón M, Flexas J, DeLucia E H. Photosynthesis responses to biotic stress. En: Terrestrial photosynthesis in a changing environment. A molecular, physiological and ecological approach. Ed. J. Flexas, F. Loreto and $\mathrm{H}$. Medrano. Cambridge:Cambridge Press; 2012. p. 331-350.

5. McElrone AJ, Forseth IN. Photosynthetic responses of a temperate liana to Xylella fastidiosa infection and water stress. J Phytopathol. 2004;152(1):9-20.

6. Berova N, Di Bari L, Pescitelli G. Application of electronic circular dichroism in configurational and conformational analysis of organic compounds. Chem Soc Rev. 2007;36(6):914-31.

7. Tao Y, Xie Z. ChenW, Glazebrook J, Chang HS, Han B, et al. Quantitative nature of Arabidopsis responses during compatible and incompatible interactions with the bacterial pathogen Pseudomonas syringae. Plant Cell. 2003;15:317-30.

8. Zou J, Rodriguez-Zas S, Aldea M, Li M, Zhu J, Gonzalez DO, et al. Expression profiling soybean response to Pseudomonas syringae reveals new defenserelated genes and rapid HR-specific downregulation of photosynthesis. MPMI. 2005;18:1161-74.

9. Truman W, Torres de Zabala M, Grant M. Type III effectors orchestrate a complex interplay between transcriptional networks to modify basal defence responses during pathogenesis and resistance. Plant J. 2006;46:14-33.

10. Jones AME, Thomas V, Bennett MH, Mansfield JW, Grant M. Modifications to the Arabidopsis defense proteome occur prior to significant transcriptional change in response to inoculation with Pseudomonas syringae. Plant Physiol. 2006;142:1603-20.

11. Ichinose $Y$, Taguchi F, Mukaihara T. Pathogenicity and virulence factors of Pseudomonas syringae. J Gen Plant Pathol. 2013;79:285-96.

12. Young JM, Takikawa Y, Gardan L, Stead DE. Changing concepts in the taxonomy of plant pathogenic bacteria. Annu Rev Phytopathol. 1992;30:67-105.

13. Mansfield J, Genin S, Magori S, Citovsky V, Sriariyanum M, Ronald P, et al. Top 10 plant pathogenic bacteria in molecular plant pathology. Mol Plant Pathol. 2012;13:614-29.

14. Bonfig KB, Schreiber U, Gabler A, Roitsch T, Berger S. Infection with virulent and avirulent $P$. syringae strains differentially affects photosynthesis and sink metabolism in Arabidopsis leaves. Planta. 2006;225(1):1-12.

15. Berger S, Benediktyová Z, Matouš K, Bonfig K, Mueller MJ, Nedbal L, et al. Visualization of dynamics of plant-pathogen interaction by novel combination of chlorophyll fluorescence imaging and statistical analysis: differential effects of virulent and avirulent strains of $P$. syringae and of oxylipins on A. thaliana. J Exp Bot. 2007;58(4):797-806.

16. Pérez Bueno ML, Pineda M, Díaz Casado E, Barón M. Spatial and temporal dynamics of primary and secondary metabolism in Phaseolus vulgaris challenged by Pseudomonas syringae. Physiol Plantarum. 2015;153(1):161-74

17. Rodríguez-Moreno L, Pineda M, Soukupová J, Macho AP, Beuzón CR, Barón M. Early detection of bean infection by Pseudomonas syringae in asymptomatic leaf areas using chlorophyll fluorescence imaging. Photosynth Res. 2008;96(1):27-35.

18. Uchytil TF, Durbin RD. Hydrolysis of tabtoxins by plant and bacterial enzymes. Experientia. 1980:36:301-2.

19. Ramegowda V, Senthil-Kumar M, Ishiga Y, Kaundal A, Udayakumar M, Mysore KS. Drought stress acclimation imparts tolerance to Sclerotinia sclerotiorum and Pseudomonas syringae in Nicotiana benthamiana. Int J Mol Sci. 2013:14(5):9497-513.

20. Lee S, Yang DS, Uppalapati SR, Sumner LW, Mysore KS. Suppression of plant defense responses by extracellular metabolites from Pseudomonas syringae pv tabaci in Nicotiana benthamiana. BMC Plant Biol. 2013;13:65.

21. Hann DR, Rathjen JP. Early events in the pathogenicity of Pseudomonas syringae on Nicotiana benthamiana. Plant J. 2007:49:607-18.

22. Taguchi F, Ichinose Y. Role of type IV Pili in virulence of Pseudomonas syringae pv. tabaci 6605: correlation of motility, multidrug resistance, and HR-inducing activity on a nonhost plant. MPMI. 2011;24(9):1001-11.

23. Aro EM, Virgin I, Andersson B. Photoinhibition of photosystem II. Inactivation, protein damage and turnover. Biochim Biophys Acta. 1993;1143:113-34.

24. Nelson N, Ben-Shem A. The complex architecture of oxygenic photosynthesis. Nat Rev Mol Cell Bio. 2004;5:1-12.

25. Rochaix JD. Assembly of photosynthetic complexes. Plant Physiol. 2011;155: 1493-500. 
26. Clavier CGJ, Boucher G. The use of photosynthesis inhibitor (DCMU) for in situ metabolic and primary production studies on soft bottom benthos. Hydrobiologia. 1992;246(2):141-5.

27. Takano S, Tomita J, Sonoike K, et al. The initiation of nocturnal dormancy in Synechococcus as an active process. BMC Biol. 2015;13(1):36.

28. Strasser BJ. Donor side capacity of photosystem II probed by chlorophyll $a$ fluorescence transients. Photosynth Res. 1997;52:147-55.

29. Tóth SZ, Schansker G, Kissimon J, Kovács L, Garab G, Strasser RJ. Biophysical studies of photosystem I-related recovery processes after a heat pulse in barley seedlings (Hordeum vulgare L.). J Plant Physiol. 2005;162:181-94.

30. Strasser BJ, Strasser RJ. Measuring fast fluorescence transients to address environmental questions: the JIP-test [M]. In: Photosynthesis: From Light to Biosphere. Ed, P Mathis. Dordrecht:Kluwer Academic Publishers; 1995. p. 977-980.

31. Li PM, Cheng LL, Gao HY, Jiang CD, Peng T. Heterogeneous behavior of PSI in soybean (Glycine max) leaves with identical PSII photochemistry efficiency under different high temperature treatments. J Plant Physiol. 2009;166: 1607-15.

32. Haldimann P, Strasser RJ. Effects of anaerobiosis as probed by the polyphasic chlorophyll a fluorescence rise kinetic in pea (Pisum sativum L.) Photosynth Res. 1999;62:67-83.

33. Fleury C, Mignotte $B$, Vayssière $J$ L. Mitochondrial reactive oxygen species in cell death signaling. Biochimie. 2002;84:131-41.

34. Meinhardt LW, Costa GGL, Thomazella DPT, Thomazella DP, Teixeira PJP, Carazzolle MF, et al. Genome and secretome analysis of the hemibiotrophic fungal pathogen, Moniliophthora roreri, which causes frosty pod rot disease of cacao: mechanisms of the biotrophic and necrotrophic phases. BMC Genomics. 2014;15(1):164.

35. Balachandran S, Osmond CB, Daley PF. Diagnosis of the earliest strainspecific interactions between tobacco mosaic virus and chloroplasts of tobacco leaves in vivo by means of chlorophyll fluorescence imaging. Plant Physiol. 1994;104(3):1059-65.

36. Balachandran S, Osmond CB, Makino A. Effects of two strains of tobacco mosaic virus on photosynthetic characteristics and nitrogen partitioning in leaves of Nicotiana tabacum cv Xanthi during photoacclimation under two nitrogen nutrition regimes. Plant Physiol. 1994;104(3):1043-50.

37. Kudoh H, Sonoike K. Irreversible damage to photosystem I by chilling in the light: Cause of the degradation of chlorophyll after returning to normal growth temperature. Planta. 2002;215:541-8.

38. Thomas MD, Langston-Unkefer PJ, Uchytil TF, Durbin RD. Inhibition of glutamine synthetase from pea by tabtoxinine- $\beta$-lactam. Plant Physiol. 1983; 71:912-5.

39. Sharkey TD, Bernacchi CJ, Farquhar GD, Singsaas EL. Fitting photosynthetic carbon dioxide response curves for C3 leaves. Plant Cell Environ. 2007;30: 1035-40.

40. Lepiniec L, Vidal J, Chollet R, Gadal P, Crétin C. Phosphoenolpyruvate carboxylase: structure, regulation and evolution. Plant Sci. 1994;99:111-24.

41. Sankhla N, Huber W. Regulation of balance between C3 and C4 pathway: role of abscisic acid. Z Pflanzenphysiol. 1974;74:267-71.

42. Amzallag GN, Lerner HR, Poljakoff-Mayber A. Exogenous ABA as a modulator of the response of sorghum to high salinity. J Exp Bot. 1990:41:1529-34.

43. Popova LP, Stoinova ZG, Maslenkova LT. Involvement of abscisic acid in photosynthetic process in Hordeum vulgare L. during salinity stress. J Plant Growth Regul. 1995;14:211-8.

44. Muller K, Doubnerova V, Synkova H, Cerovska N, Ryslava H. Regulation of phosphoenolpyruvate carboxylase in PVYNTN-infected tobacco plants. Bio Chem. 2009;390:245-51.

45. Ryslava H, Muller K, Semoradova S, Synkova H, Cerovska N. Photosynthesis and activity of phosphoenolpyruvate carboxylase in Nicotiana tabacum L. leaves infected by Potato virus A and Potato virus Y. Photosynthetica. 2003; 41:357-63.

46. Tietz S, Wild A. Investigations on the phosphoenolpyruvate carboxylase activity of spruce needles relative to the occurrence of novel forest decline. Plant Physiol. 1991;137:327-31.

47. Goh CH, Ko SM, Koh S, Kim YJ, Bae HJ. Photosynthesis and environments: photoinhibition and repair mechanisms in plants. J Plant Biol. 2012;55:93-101.

48. Barth C, Krause GH, Winter K. Responses of photosystem I compared with photosystem II to high-light stress in tropical shade and sun leaves. Plant Cell Environ. 2001;24:163-76.

49. Öquist G, Huner NPA. Photosynthesis of overwintering plants. Annu Rev Plant Biol. 2003;54:329-55.
50. Terashima I, Funayama S, Sonoike K. The site of photoinhibition in leaves of Cucumis sativus L. at low temperatures is photosystem I, not photosystem II. Planta. 1994;193:300-6.

51. Asada K. The water-water cycle in chloroplasts: scavenging of active oxygens and dissipation of excess photons. Annu Rev Plant Biol. 1999;50(1):601-39.

52. Galvez Valdivieso G, Mullineaux PM. The role of reactive oxygen species in signalling from chloroplasts to the nucleus. Physiol Plantarum. 2010;138(4): 430-9.

53. Apel K, Hirt H. Reactive oxygen species: metabolism, oxidative stress, and signal transduction. Annu Rev Plant Biol. 2004;55:373-99.

54. Allakhverdiev SI, Murata N. Environmental stress inhibits the synthesis de novo of proteins involved in the photodamage-repair cycle of Photosystem II in Synechocystis sp. PCC 6803. Biochim Biophys Acta. 2004;1657:23-32.

55. Parrado J, Absi EH, Machado A, Ayala A. In vitro effect of cumene hydroperoxide on hepatic elongation factor-2 and its protection by melatonin. Biochim Biophys Acta. 2003;1624:139-44.

56. Murata N, Takahashi S, Nishiyama Y, Allakhverdiev SI. Photoinhibition of photosystem II under environmental stress. BBA-Bioenergetics. 2007;1767(6): 414-21.

57. Takahashi S, Murata N. How do environmental stresses accelerate photoinhibition? Trends Plant Sci. 2008;13:178-82.

58. Sonoike K, Kamo M, Hihara Y, Hiyama T, Enami I. The mechanism of the degradation of psaB gene product, one of the photosynthetic reaction center subunits of photosystem I upon photoinhibition. Photosynth Res. 1997;53:55-63.

59. Fan X, Zhang Z, Gao H, Yang C, Liu M, Li Y, et al. Photoinhibition-like damage to the photosynthetic apparatus in plant leaves induced by submergence treatment in the dark. PLoS One. 2014;9:e89067.

60. de Torres Zabala M, Littlejohn G, Jayaraman S, Studholme D, Bailey T, Lawson T, et al. Chloroplasts play a central role in plant defence and are targeted by pathogen effectors. Nature Plants. 2015;1(6):1-10.

61. Yamori W, Noguchi KO, Hikosaka K, Terashima I. Phenotypic plasticity in photosynthetic temperature acclimation among crop species with different cold tolerances. Plant Physiol. 2010;152:388-99.

62. Nishiyama Y, Allakhverdiev SI, Murata N. Protein synthesis is the primary target of reactive oxygen species in the photoinhibition of photosystem II. Physiol Plant. 2011;142:35-46.

63. Balachandran S, Osmond CB. Susceptibility of tobacco leaves to photoinhibition following infection with two strains of tobacco mosaic virus under different light and nitrogen nutrition regimes. Plant Physiol. 1994; 104(3):1051-7.

64. King EO, Wood MK, Raney DE. Two simple media for the demonstration of pyocyanin and fluorescin. J Lab Clin Med. 1954:44(2):301-7.

65. Porra RJ. The chequered history of the development and use of simultaneous equations for the accurate determination of chlorophylls a and b. Photosynth Res. 2002;73:149-56.

66. Bingham M J, Long S P. Equipment for field and laboratory studies of whole plant and crop photosynthesis and productivity research. Techniques in Bioproductivity and Photosynthesis: Pergamon International Library of Science, Technology, Engineering and Social Studies, 2014. p. 229

67. Strasser RJ, Tsimilli-Michael M, Qiang S, Goltsev V. Simultaneous in vivo recording of prompt and delayed fluorescence and 820-nm reflection changes during drying and after rehydration of the resurrection plant Haberlea rhodopensis. BBA-Bioenergetics. 2010;1797(6):1313-26.

68. Gao J, Li P, Ma F, Goltsev V. Photosynthetic performance during leaf expansion in Malus micromalus probed by chlorophyll a fluorescence and modulated $820 \mathrm{~nm}$ reflection. J Photoch Photobio B. 2013;137:144-50.

69. Oukarroum A, Goltsev V, Strasser RJ. Temperature effects on pea plants probed by simultaneous measurements of the kinetics of prompt fluorescence, delayed fluorescence and modulated $820 \mathrm{~nm}$ reflection. PLoS One. 2013;8:e59433.

70. Patterson BD, Macrae EA, Ferguson IB. Estimation of hydrogen peroxide in plant extracts using titanium (IV). Anal Biochem. 1984;139:487-92. 